\title{
MPS1 kinase as a potential therapeutic target in medulloblastoma
}

\author{
IRINA ALIMOVA $^{1 *}$, JUNE NG $^{2 *}$, PETER HARRIS $^{1}$, DIANE BIRKS ${ }^{3}$, ANDREW DONSON $^{1}$, \\ MICHAEL D. TAYLOR ${ }^{4}$, NICHOLAS K. FOREMAN ${ }^{1,3,5}$, SUJATHA VENKATARAMAN ${ }^{1}$ and RAJEEV VIBHAKAR RI, $^{1,3,5}$
}

\author{
${ }^{1}$ Department of Pediatrics, University of Colorado Denver, Anschutz Medical Campus; ${ }^{2}$ University of Colorado School of \\ Medicine, Anschutz Medical Campus; ${ }^{3}$ Department of Neurosurgery, University of Colorado Denver, Anschutz Medical \\ Campus, Aurora, CO 80045, USA; ${ }^{4}$ Division of Neurosurgery, Program in Developmental and Stem Cell Biology, \\ Hospital for Sick Children, Toronto, ON M5G 1X8, Canada; ${ }^{5}$ Children's Hospital Colorado, Aurora, CO 80045, USA
}

Received February 4, 2016; Accepted May 27, 2016

DOI: $10.3892 / o r .2016 .5085$

\begin{abstract}
Medulloblastoma is the most common type of malignant brain tumor that affects children. Although recent advances in chemotherapy and radiation have improved outcomes, high-risk patients perform poorly with significant morbidity. Gene expression profiling has revealed that monopolar spindle 1 (MPS1) (TTK1) is highly expressed in medulloblastoma patient samples compared to that noted in normal cerebellum. MPS1 is a key regulator of the spindle assembly checkpoint (SAC), a mitotic mechanism specifically required for proper chromosomal alignment and segregation. The SAC can be activated in aneuploid cancer cells and MPS1 is overexpressed in many types of cancers. A previous study has demonstrated the effectiveness of inhibiting MPS1 with small-molecule inhibitors, but the role of MPS1 in medulloblastoma is unknown. In the present study, we demonstrated that MPS1 inhibition by shRNA or with a small-molecule drug, NMS-P715, resulted in decreased cell growth, inhibition of clonogenic potential and induction of apoptosis in cells belonging to both the Shh and group 3 medulloblastoma genomic signature. These findings highlight MPS1 as a rational therapeutic target for medulloblastoma.
\end{abstract}

\section{Introduction}

Medulloblastoma is the most common malignant brain tumor in children. Patients are risk stratified according to age (greater or less than 3 years of age), presence of disease dissemination and extent of resection. The mainstays of therapy are surgery, chemotherapy and irradiation. However, all of these therapies result in long-term problems (1). Although a variety

Correspondence to: Dr Rajeev Vibhakar, Department of Pediatrics, University of Colorado Denver, Anschutz Medical Campus, 12800 19th Avenue, Aurora, CO 80045, USA

E-mail: rajeev.vibhakar@ucdenver.edu

${ }^{*}$ Contributed equally

Key words: medulloblastoma, MPS1, mitosis, NMS-P715, kinase of signaling pathways [(e.g. Sonic hedgehog (SHH) signaling, Wnt signaling (WNT)] are known to be associated with medulloblastoma cell biology (2-4), new therapeutic interventions for medulloblastoma have been slow to develop. Recent genomic analyses have identified multiple molecular subgroups with differing outcomes $(5,6)$, underscoring the heterogeneity of medulloblastoma. By current international consensus there are four main subgroups of medulloblastoma: WNT, SHH, groups 3 and 4 (7). However these molecular data are not yet used to guide therapy (8). Therefore, there is a critical need for new treatment agents for medulloblastoma.

We recently identified several kinases involved in the G2/M cell cycle checkpoint that influence medulloblastoma cell viability (9). Among these is the monopolar spindle 1 (MPS1) kinase, which is widely conserved amongst eukaryotes. MPS1 is involved, along with Aurora-B kinase, in ensuring proper orientation of sister chromatids at the kinetochore during the spindle assembly checkpoint (SAC) as well as activation and maintenance of the checkpoint itself (10). Additionally, overexpression of mammalian Mps1 leads to overduplication of the centrosome, and conversely expression of the kinase-inactive allele prevents centrosome duplication (11).

MPS1 is often dysregulated in cancers such as thyroid papillary carcinoma, breast, gastric and lung cancer, suggesting that increased expression of MPS1 may either promote cancer initiation or allow survival of aneuploidy cancer cells (10). Recently, studies have shown that inhibition of MPS1 induces aberrant mitosis and apoptosis in a variety of cancer cells including human breast cancer cells $(12,13)$. In cervical carcinoma cells inhibition of MPS1 by a novel selective small-molecule inhibitor, NMS-P715, led to SAC override and mitotic acceleration (14). In ovarian cancer and colon carcinoma cells, NMS-P715 treatment also led to aneuploidy and apoptosis (14). NMS-P715 showed significant antiproliferative activity in colon, breast, renal and melanoma cancer cell lines, but did not alter proliferation of normal non-transformed cells (14). However, the role of MPS1 in medulloblastoma has yet to be evaluated.

In the present study, we showed that medulloblastoma patient samples expressed higher levels of MPS1 compared to that noted in normal cerebellum. Inhibition of MPS1 suppressed medulloblastoma cell growth and treatment of medulloblastoma cells with NMS-P175 suppressed medulloblastoma colony formation and induced apoptosis. 


\section{Materials and methods}

Cell lines and reagents. The Daoy and D283 medulloblastoma cell lines were purchased from the American Type Culture Collection (ATCC) (Rockville, MD, USA). The ONS-76 medulloblastoma cell line was provided by Dr James T. Rutka at the University of Toronto, Canada, and the UW228 cell line by Dr John Silber at the University of Washington (Seattle, WA, USA). D425 and D458 cell lines were obtained from Dr Darell D. Bigner at Duke University Medical Center (Durham, NC, USA). Cell lines were cultured in Dulbecco' modified Eagle's medium (DMEM) (Gibco, Carlsbad, CA, USA) supplemented with $10 \%$ fetal bovine serum (FBS) (Atlanta Biologicals, Lawrenceville, GA, USA). Cell lines D283, D425 and D458 are all part of the group 3 genomic subgroup. Daoy, ONS-76 and UW228 cluster with the Shh subgroup.

Primary patient samples were obtained from Children's Hospital Colorado and were collected and used in accordance with local and federal human research protection guidelines and Institutional Review Board (IRB) regulations. Informed consent was obtained for all specimens collected. Normal brain tissue was collected from autopsy and purchased from Ambion (Austin, TX, USA), Stratagene (Santa Clara, CA, USA) and Clontech Laboratories, Inc. (Mountain View, CA, USA). NMS-P715 was kindly provided by R. Colombo, Nerviano Medical Sciences (Nerviano, Italy) or were purchased from Calbiochem, EMD Millipore Corp. (Billerica, MA, USA). NMS-P715 was reconstituted in dimethyl sulfoxide (DMSO) and aliquots were stored in a desiccator at $-20^{\circ} \mathrm{C}$. An equivalent amount of DMSO for the highest concentration of drug was used for each experiment as a vehicle control.

Gene expression microarray analysis. Patient tumor samples were collected at the time of surgery and snap-frozen in liquid nitrogen. Ribonucleic acid was extracted from all samples using an RNeasy kit (Qiagen, Valencia, CA, USA). Samples were evaluated for gene expression using Affymetrix U133 Plus 2.0 GeneChip microarrays as we previously described $(9,15)$.

Cell proliferation assay. Cell proliferation was determined by 3-(4,5-dimethylthiazol-2-yl)-5-(3-carboxymethoxyphenyl)2-(4-sulfophenyl)-2H-tetrazolium (MTS) assay using CellTiter 96 AQueous One Solution (Promega, Madison, WI, USA). Seventy-two hours after transfection with shTTK1 (shMPS1), $20 \mu 1$ of MTS reagent was added to the wells already containing $100 \mu 1$ of media.

For drug treatment, the cells were plated for $24 \mathrm{~h}$ before adding 0.5 and $2 \mu \mathrm{m}$ of NMS-P715. Then, $72 \mathrm{~h}$ after the addition of the drug, $30 \mu \mathrm{l}$ of MTS reagent was added to the wells to make a final volume of $180 \mu \mathrm{l}$. Plates were read using a Bio-Tek Synergy 2 plate reader (Bio-Tek, Winooski, VT, USA) every hour for $4 \mathrm{~h}$ after the addition of the MTS reagent. Experiments were carried out in triplicate and background absorbance was subtracted from all wells before analysis.

Viability cell assay. Cell numbers after NMS-P715 treatment $(2 \mu \mathrm{m})$ were measured using the ViaCount assay (Millipore) on a Guava flow cytometer as per the manufacturer's recommendations. Cells were cultured for $72 \mathrm{~h}$, and collected by centrifugation. The cells were washed, ViaCount reagent was added, and cell counts were measured on the Guava EasyCyte Plus flow cytometer (Millipore).

Colony formation assay. Daoy, ONS-76 and UW228 medulloblastoma cells were seeded into 6-well plates in triplicate at a density of 500 cells/well in $3 \mathrm{ml}$ medium containing $10 \%$ FBS for $24 \mathrm{~h}$. The cells were then treated with DMSO or MPS1 inhibitor, NMS-P715 (500 nm-1 $\mu \mathrm{m})$ and further cultured for 10 days in a $37^{\circ} \mathrm{C}$ humidified atmosphere containing $95 \%$ air and $5 \% \mathrm{CO}_{2}$. After 10 days of growth, the medium was aspirated, the wells were washed with phosphate-buffered saline (PBS), and cell clones were stained for $15 \mathrm{~min}$ with a solution containing $0.5 \%$ crystal violet and $25 \%$ methanol, followed by 3 rinses with tap water to remove excess dye. The colony numbers were counted using a precise electronic counter (Heathrow Scientific, Vernon Hills, IL, USA) and an inverted microscope with a threshold of 50 cells necessary to constitute a colony.

Western blotting. Protein expression levels were determined by western blotting. Cells were lysed in 1X RIPA buffer (Thermo Scientific, Rockford, IL, USA) containing protease inhibitor cocktail (Roche, Indianapolis, IN, USA), $1 \mathrm{mM}$ sodium vanadate and $0.1 \mathrm{mM}$ sodium molybdate. The lysates were centrifuged for $20 \mathrm{~min}$ and the supernatants collected for protein concentration determination by the Bradford reagent (Sigma, St. Louis, MO, USA). Equal amounts of cell lysates were resolved by sodium dodecyl sulfate-polyacrylamide gel electrophoresis (SDS-PAGE), and western blot analysis was performed with specific antibodies. Antibodies used for western blot analysis were purchased from the following sources: actin \#8H10D10, MPS1\#11108 (Abcam, Cambridge, MA, USA). Antibodies for Mps1 and phosphorylated Mps1 were obtained by P.A. Eyers (16). Secondary antibodies conjugated to horseradish-peroxidase were used in conjunction with a chemiluminescent reagent to visualize protein bands.

Cell cycle assay. Flow cytometric analysis was performed to define the cell cycle distribution after NMS-P715 treatment. D425 and D458 cells were seeded into 6-well plates $\left(10^{5}\right.$ cells/well) and $24 \mathrm{~h}$ later were treated with $2 \mu \mathrm{m}$ NMS-P715. Cells were harvested $72 \mathrm{~h}$ later and fixed with chilled $70 \%$ ethanol for $24 \mathrm{~h}$. Fixed cells were then washed and stained with propidium iodide-containing cell cycle reagent (Millipore). Flow cytometric analysis was performed on the Guava EasyCyte Plus flow cytometer.

Cell apoptosis assay. Daoy and UW228 medulloblastoma cells were treated with $2 \mu \mathrm{m}$ NMS-P715 and allowed to grow in normal culture medium for $72 \mathrm{~h}$. The cell concentration was determined following staining with Guava ViaCount reagent. Equal numbers of cells were then stained using Guava Nexin reagent (Millipore) to detect apoptotic cells. Samples were run on a Guava EasyCyte Plus flow cytometer.

\section{Results}

MPS1 is highly expressed in high grade pediatric brain tumors. Our recent study suggested that MPS1 is highly expressed in medulloblastoma (9). To further examine the expression of MPS1 in pediatric brain tumors we first 


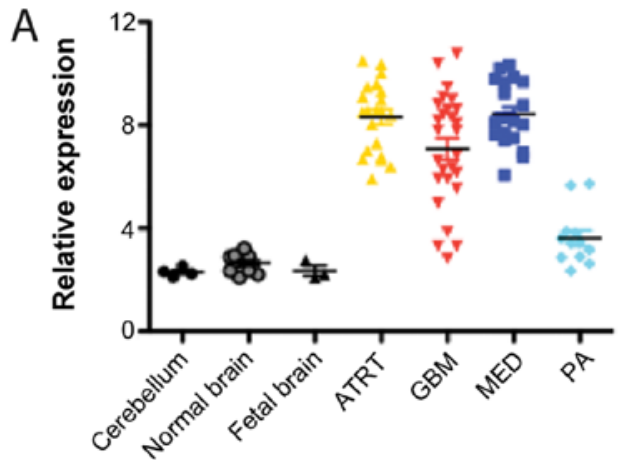

C

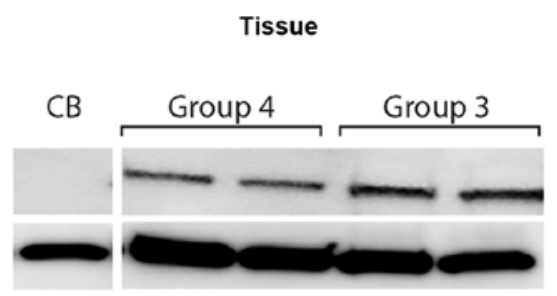

B

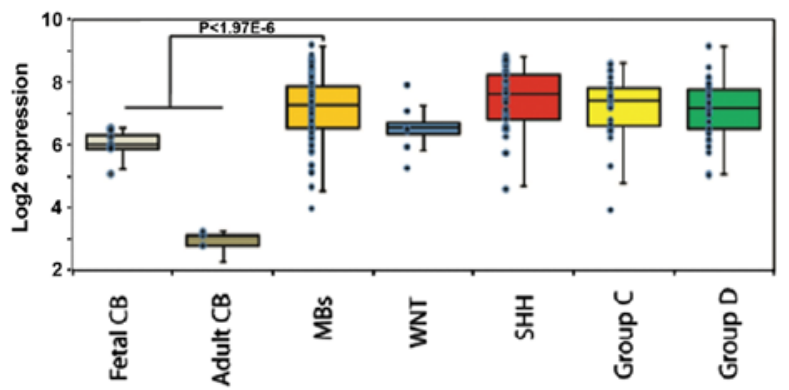

D

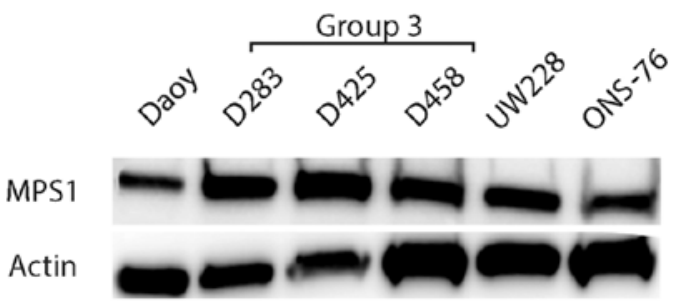

Figure 1. Overexpression of MPS1 in pediatric brain tumors. (A) Microarray expression of MPS1 mRNA in 90 pediatric brain tumors showed significant overexpression of MPS1 in high-grade pediatric brain tumors when compared to normal brain. Error bars represent standard error of the mean (SEM). (B) Microarray expression of MPS1 mRNA in 120 patients with medulloblastoma demonstrated that MPS1 mRNA is significantly elevated in medulloblastoma compared to adult and fetal cerebellum but not significantly different among the genomic subgroups (WNT, SHH, groups 3 and 4). Error bars represent SEM. (C) MPS1 protein expression in patient tumor samples including two group 3 and two group 4 samples compared to normal pediatric cerebellum (CB). (D) MPS1 protein expression in commonly used medulloblastoma cell lines (D283, D458 and D425, group 3 genomic subgroup; Daoy, ONS-76 and UW228, Shh genomic subgroup). ATRT, atypical teratoid/rhabdoid tumor: GMB, glioblastoma multiforme; MED, medulloblastoma; PA, pilocytic astrocytoma.

analyzed microarray-derived gene expression data from 90 pediatric brain tumors. MPS1 mRNA expression was significantly elevated in high grade pediatric brain tumors including glioblastoma (GBM), medulloblastoma (MED) and ATRT compared to normal brain (Fig. 1A). Notably, lower grade tumors such as pilocytic astrocytoma (PA) expressed much lower amounts of MPS1 mRNA (Fig. 1A). There was no clear correlation between the high MPS1-expressing samples and age, gender or outcomes.

Medulloblastoma consists of four major genomic subgroups [Sonic hedgehog (SHH) signaling, Wnt signaling (WNT), and groups 3 and 4 (7)]. To further elucidate whether there was a correlation between the subgroups of medulloblastoma, we examined expression of MPS1 mRNA in a cohort of 120 recently described medulloblastoma samples (5). MPS1 mRNA was significantly elevated in medulloblastoma compared to adult and fetal cerebellum (Fig. 1B). However, MPS1 expression was not significantly different in any of the medulloblastoma genomic subgroups (Fig. 1B). Similarly, MPS1 protein expression was elevated in the tumor samples from groups 3 and 4 patients compared to normal cerebellum (Fig. 1C). All group 3 and Shh medulloblastoma cell lines commonly used also demonstrated significantly elevated MPS1 protein expression (Fig. 1D). These data suggest that MPS1 may be associated with the oncogenic process in general and is not specific to a particular molecular subgroup of medulloblastoma.

Inhibition of MPS1 suppresses medulloblastoma cell growth. We hypothesized that overexpression of MPS1 drives medulloblastoma cell growth and that inhibition of MPS1 would repress medulloblastoma cell growth. To test this hypothesis we first suppressed MPS1 mRNA in 2-well characterized cell lines using shRNA. In both Daoy and ONS-76 cell lines transfection of shTTK1 (shMPS1) significantly decreased the number of viable cells after $72 \mathrm{~h}$ as measured by the MTS assay (Fig. 2A and B). Knockdown of MPS1 was verified by qRT-PCR ( $43 \%$ decrease in MPS1 mRNA compared to sh-control, data not shown).

To further evaluate the impact of MPS1 inhibition we obtained a recently described MPS1 inhibitor NMS-P175 (kind gift from Nervino Medical Sciences). NMS-P715 is an orally bioavailable, potent and selective small-molecule inhibitor of MPS1 kinase activity (14).

NMS-P175 potently inhibited the growth of both Daoy and ONS-76 cells in a dose- and time-dependent manner (Fig. 2C and D). Since both Daoy and ONS-76 cell lines belong to the Shh genomic group we chose to test the impact of chemical inhibition of MPS1 in additional cell lines. We chose 3 cell lines with Myc translocations that are known to be part of the group 3 medulloblastoma genomic signature. The growth of all 3 Myc cell lines (D283, D425 and D458) was also potently suppressed by MPS1 inhibition using NMS-P175 (Fig. 2E).

To evaluate a longer-term impact, we then performed colony formation assays on cells treated with varying concentrations of NMS-P715. We used lower concentrations of NMS-P715 due to the longer-term treatment. Once again, inhibition of MPS1 significantly decreased medulloblastoma cell growth as measured by their ability to form colonies (Fig. 3). Notably, in the ONS-76 cells there was a much more marked inhibition in the colony formation capability (despite treatment with a lower dose) compared to measuring just cell proliferation 

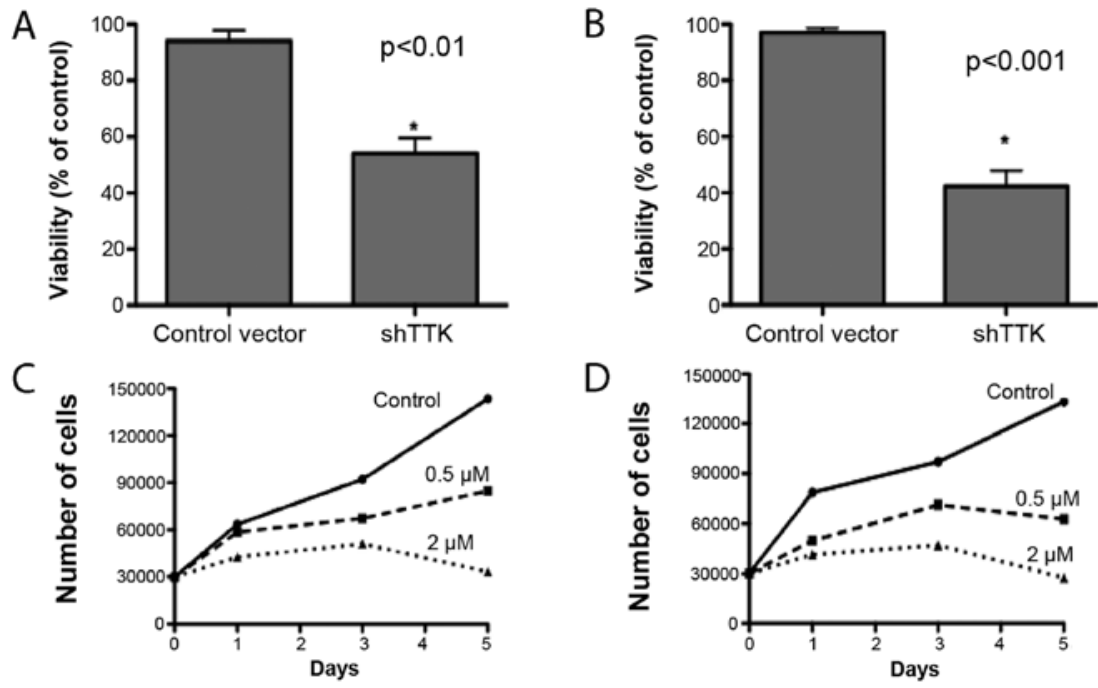

E

D283

D425
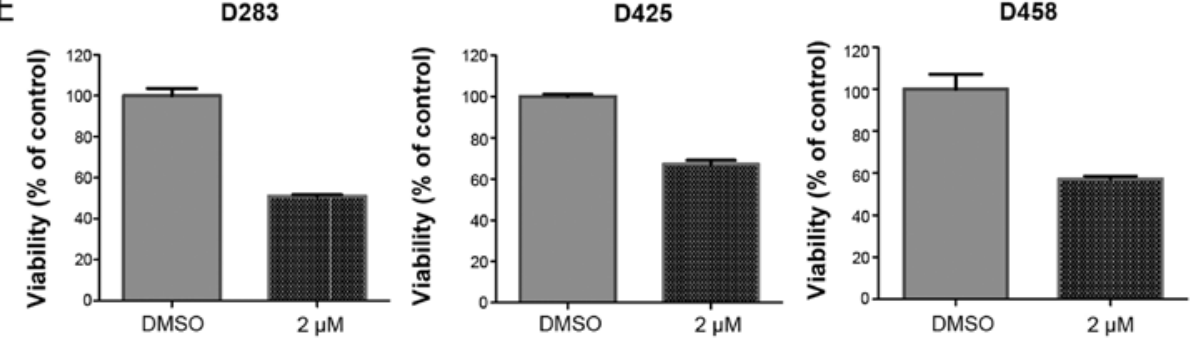

Figure 2. Inhibition of MPS1 suppresses medulloblastoma cell growth in vitro. Knockdown of MPS1 mRNA with shTTK significantly decreased the number of viable cells in both (A) Daoy and (B) ONS-76 cell lines as measured by MTS assay. Inhibition of medulloblastoma cell growth by MPS1 kinase inhibitor, NMS-P715 in a concentration-dependent manner in the (C) Daoy and (D) ONS-76 cells. (E) Activity of NMS-P715 (2 $\mu$ M) against a panel of Myc-driven medulloblastoma cell lines. All cells were treated for $72 \mathrm{~h}$ and each study was repeated 3 times.

A

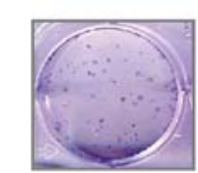

Daoy
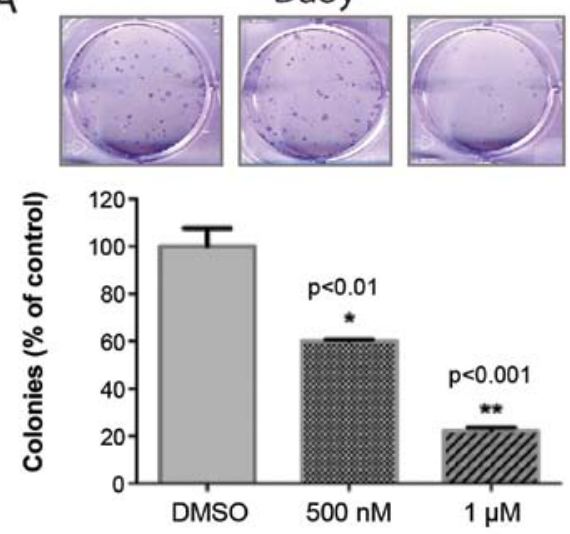

C
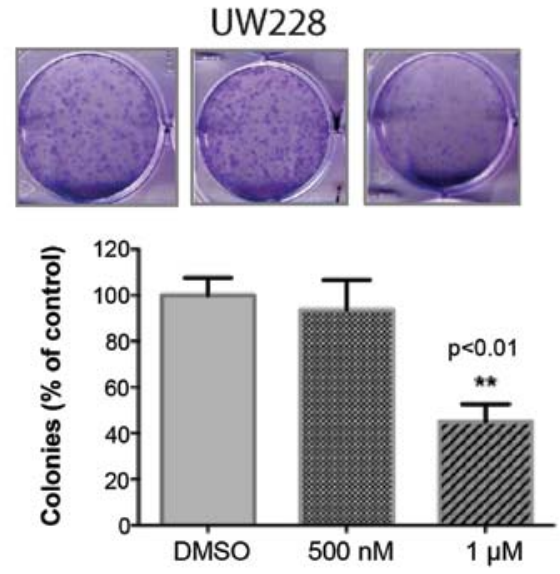

B
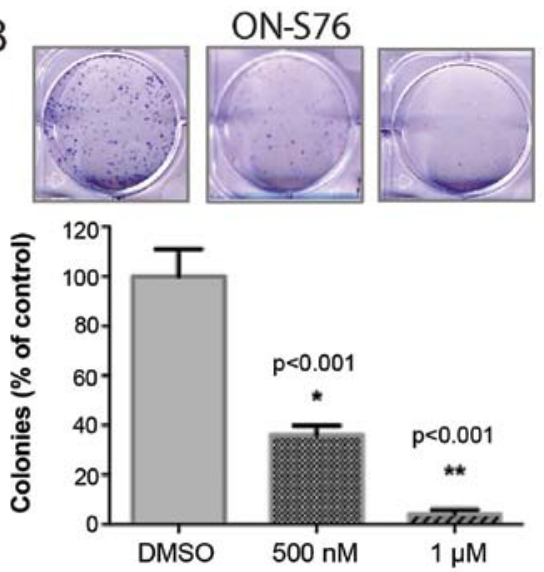

Figure 3. NMS-P715 inhibits clonogenic potential of (A) Daoy, (B) ONS-76 and (C) UW228 medulloblastoma cells. Quantification of colony formation with representative wells in control and NMS-P715-treated cells ( $\mathrm{P}<0.01$, $\left.{ }^{* *} \mathrm{P}<0.001\right)$. Cells were treated for 10 days. Error bars represent SEM. 
A

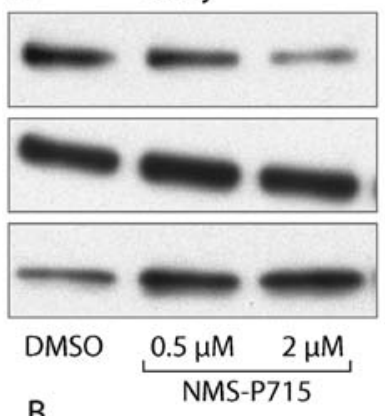

B

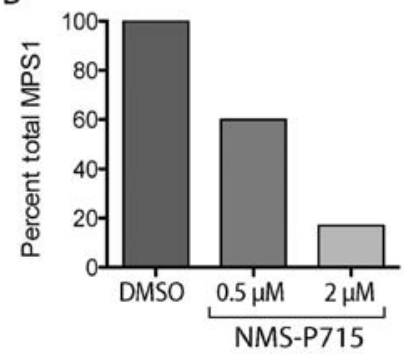

UW228

pS821

MPS1
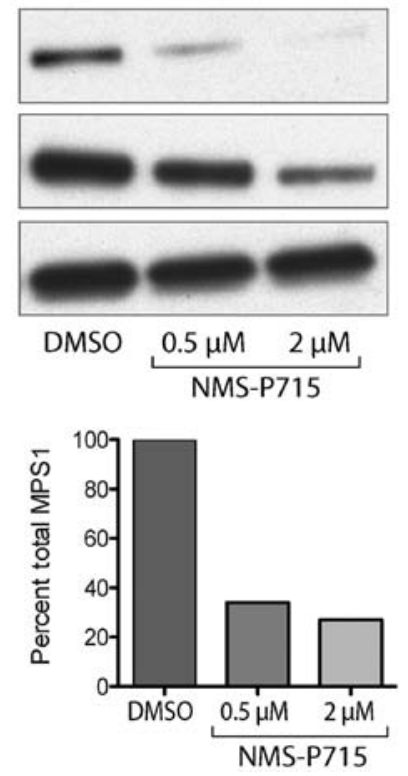

Figure 4. (A) Western blotting of NMS-P715-treated medulloblastoma cells, showing that NMS-P715 strongly decreased autophosphorylation of serine 821 on MPS1 in the Daoy and UW228 medulloblastoma cells. (B) The suppression of phosphorylation was dose-dependent for both cell lines as quantified by densitometry (pMPS1 levels relative to total MPS1 protein).

(92\% inhibition vs. $46 \%$ inhibition, respectively). These data may reflect the impact of MPS1 on tumor cell self-renewal compared to just mitosis.

NMS-P715 suppresses kinase activity of MPS1 and decreases MPS1 autophosphorylation. We further examined the functional activity of MPS1 inhibition in medulloblastoma cells. Recent evidence suggests that MPS1 phosphorylates a wide range of proteins including itself (16). To verify that inhibition of MPS1 by NMS-P715 inhibited its kinase activity, we evaluated MPS1 autophosphorylation using protein immunoblotting. In both Daoy and UW228 medulloblastoma cells NMS P715 strongly decreased the phosphorylation of serine 821 a key autophosphorylation site on MPS1 (Fig. 4A) (16). The suppression of phosphorylation was dose-dependent for both cell lines (Fig. 4B). Total MPS1 did not change in the Daoy cells, but there was a decrease in total MPS1 protein in the UW228 cells suggesting that pS821 on MPS1 may be important for protein stability.

MPS1 kinase inhibitor NMS-P715 decreases medulloblastoma cell growth by perturbing the cell cycle and increasing apoptosis. To determine whether the decreased cell growth upon MPS1 inhibition was due to cell cycle effects, we examined the impact of NMS-P715 on the cell cycle distribution of medulloblastoma cells. In all cells tested, NMS-P715 induced a G2-M arrest as shown in Fig. 5A. These data are consistent with previous studies showing that MPS1 is a critical regulator of mitosis. Moreover, western blot analysis showed that MPS1 also decreased expression of G2-M-associated proteins CDC2 and cyclin B1 (Fig. 5B).

We next examined whether apoptosis was a contributor to medulloblastoma cell growth inhibition by NMS-P715. We measured Annexin V expression on the surface of
NMS-P715-treated medulloblastoma cells by flow cytometry. Representative plots are shown for Daoy, UW228, D425 and D458 cells. Annexin V-positive-7-aminoactinomycin D (7-AAD)-negative cells, indicative of early apoptosis, were present at low levels in DMSO control-treated cells. This population increased with increasing doses of NMS-P715 (Fig. 6). In addition, the Annexin V-positive-7AAD-positive population was significantly enhanced in the NMS-P715-treated cells, indicating increased late apoptosis. The total percentage of apoptosis is quantified in Fig. 6. These data confirm that MPS1 inhibition by NMS-P715 is a strong inducer of apoptosis in medulloblastoma.

\section{Discussion}

Therapy-associated side-effects in medulloblastoma have led to a concentrated search for novel biologically based therapeutic targets (17). Given that cell cycle kinases are key regulators of tumor progression we hypothesized that these kinases are critical for medulloblastoma tumorigenesis (18). Analysis of protein kinase gene expression revealed that expression of multiple protein kinases that are components of the mitotic machinery such as Aurora kinase A, PLK1, WEE1 and now MPS1 are significantly deregulated in medulloblastoma $(9,19,20)$. Perturbing mitosis by disrupting the proper formation of mitotic spindles required for chromosome alignment and segregation has been shown to preferentially kill cancer cells (13).

MPS1 is a serine threonine kinase that regulates the mitotic spindle by triggering the spindle assembly checkpoint (SAC) (10). MPS1 plays an important role in safeguarding proper chromosome alignment and segregation during mitosis (14). Increased MPS1 levels have been found in numerous adult types of cancers, including breast, pancreatic 
A

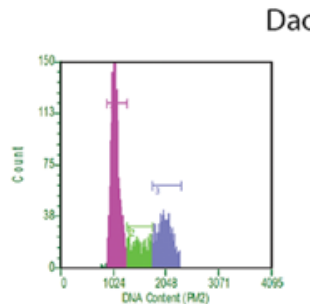

Daoy

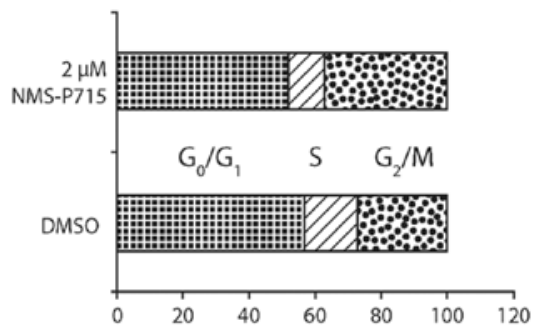

D425
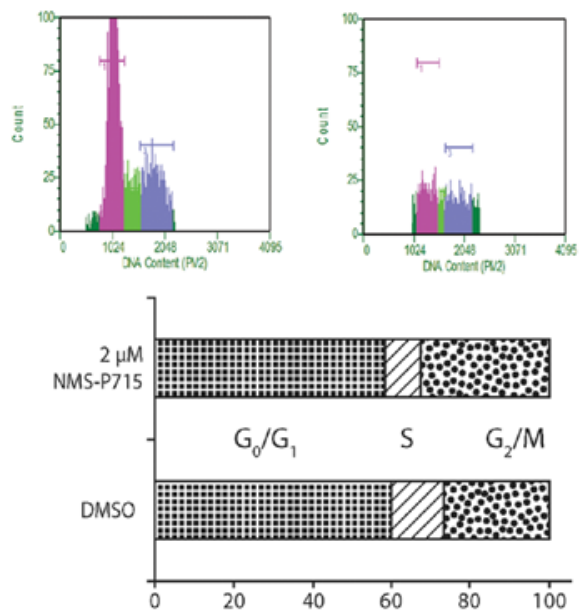

UW228
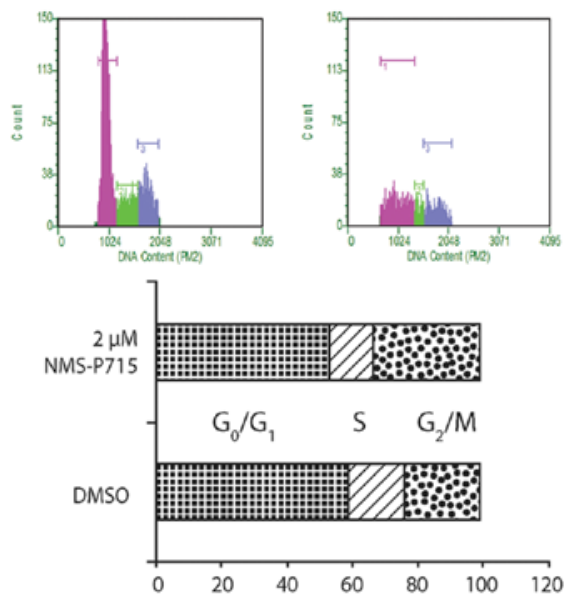

D458
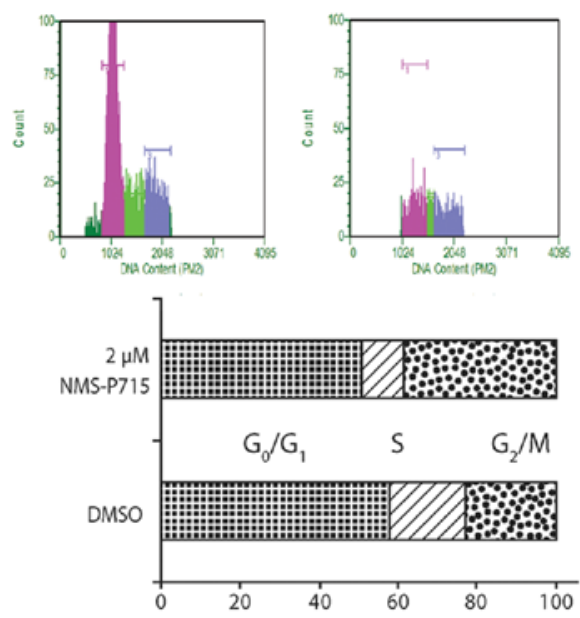

B

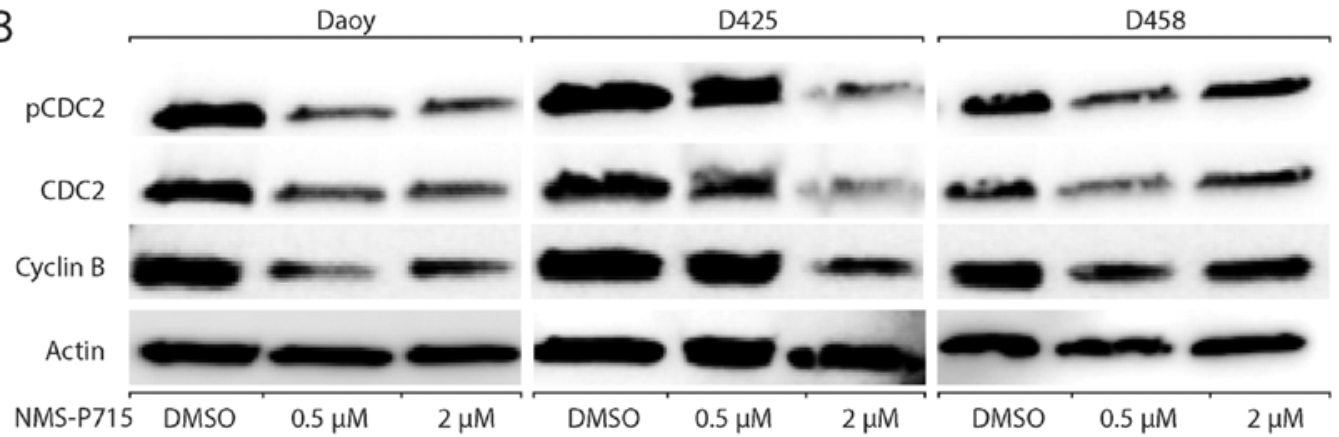

Figure 5. Chemical inhibition of MPS1 in medulloblastoma induces cell cycle arrest. (A) NMS-P715 induced G2/M arrest in medulloblastoma cells. Representative flow plots with quantification of cell cycle fractions. (B) Western blot analysis of cell cycle-related proteins upon MPS1 inhibition with NMS-P715. MPS1 inhibition resulted in decreased cyclin B2 as well as decreased CDC2 protein levels in the Daoy and D425 cells, but not in the D458 cells.

and colorectal cancer. However, the significance of MPS1 in the pathogenesis and management of medulloblastoma is not well understood.

In the present study, we demonstrated that MPS1 mRNA is overexpressed in two independent medulloblastoma cohorts when compared to normal cerebellum. Decreasing the expression of MPS1 mRNA by RNAi clearly resulted in growth suppression. Furthermore, we showed that inhibition of MPS11 by a small-molecule inhibitor, NMS-P715, resulted in a significant reduction in the proliferation of medulloblastoma cells both in short-term and long-term assays. Importantly, we showed that induction of apoptosis is a key mechanism of NMS-P715 in medulloblastoma cells.

Notably, a recent study by Maachani et al demonstrated that MPS1 is a promising molecular target in the treatment of another brain tumor, glioblastoma multiforme (GBM) (21). They showed that MPS1 inhibition can be combined with radiation to make GBM therapy more efficacious (21). In addition, several newer MPS1 inhibitors have been recently described $(10,22)$. Thus, our data along with previous studies, 
Daoy
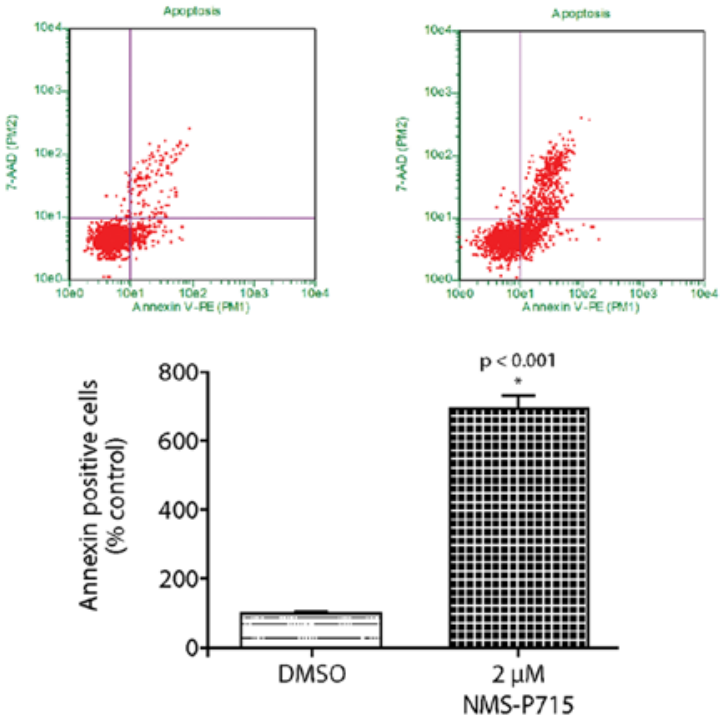

D425
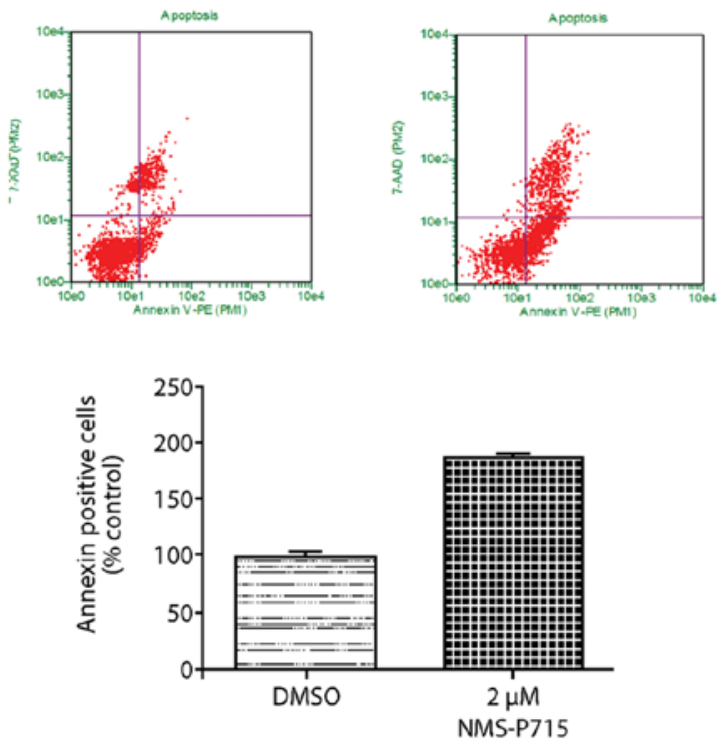

UW228
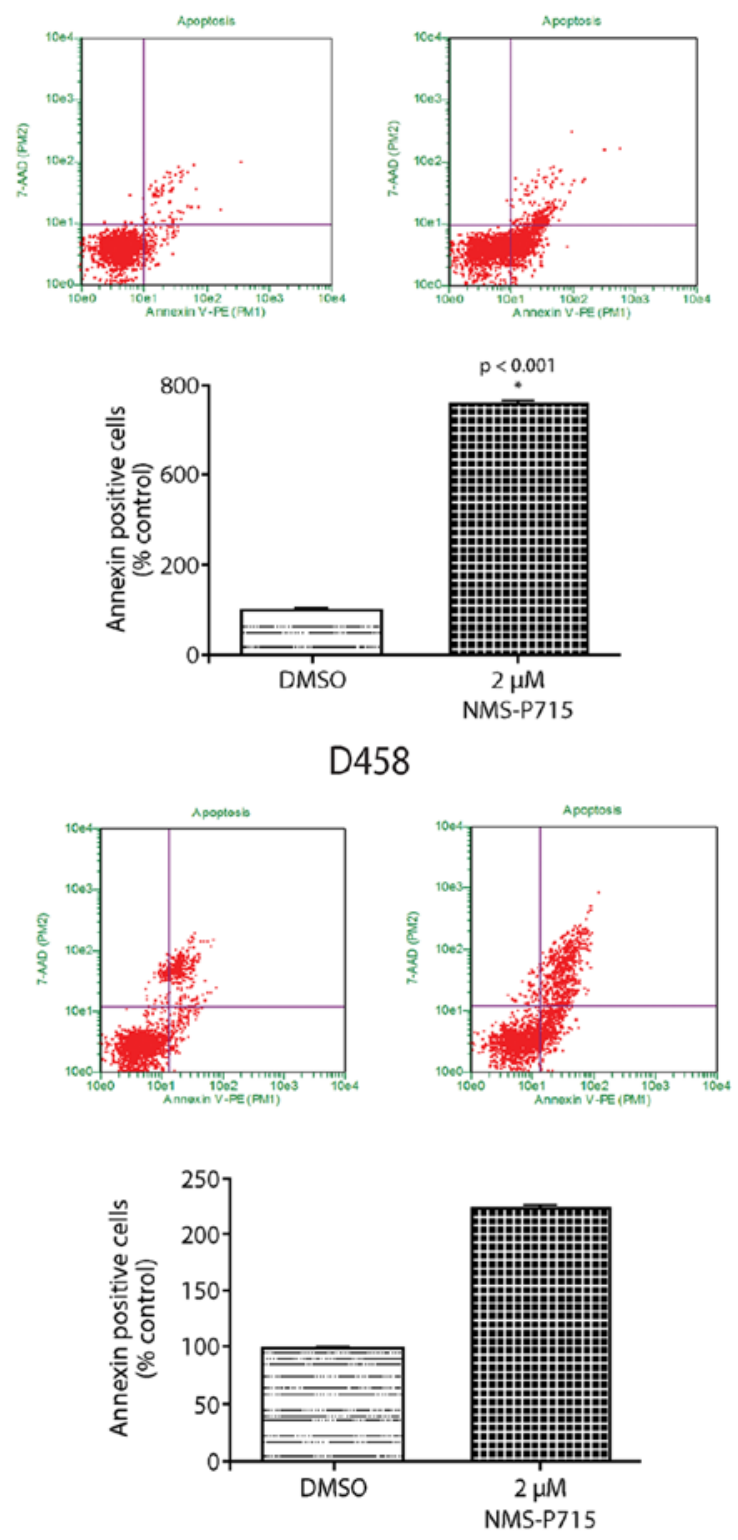

Figure 6. Inhibition of MPS1 induces apoptosis in medulloblastoma cells. Representative flow plots of Annexin V expression and quantification of apoptosis in the control and NMS-P715-treated cells.

strongly suggest that targeting MPS1 with small-molecule inhibitors is both a novel strategy in the treatment of medulloblastoma and one that warrants further study. In particular, evaluation of resistance to MPS1 inhibitors will have to be closely examined in medulloblastoma cells (23). Recent data suggest that cancer cells acquire resistance by developing point mutations in the ATP binding pocket but that the tumor cells do not develop cross resistance to other MPS1 inhibitors (23). This leaves open the possibility of using multiple MPS1 inhibitors in combinatorial and or sequential fashion. The next important step may be to test the effects of NMS-P715 in orthotopic xenograft models of medulloblastoma. It may be important to determine whether NMS-P715 offers a superior therapeutic index over current treatments, and if it can be combined with other standard treatments to improve medulloblastoma therapy.

\section{Acknowledgements}

The present study was supported by the Childhood Brain Tumor Foundation (R.V.), Department of Pediatrics, the University of Colorado School of Medicine, NIH-NINDS grants K08NS059790 and RO1NS086956 (R.V.), the Childhood Brain Tumor Foundation (R.V.), and the Morgan Adams Foundation grants (R.V. and N.K.F.). We thank Nervino Medical Sciences for the gift of the NMS-P715. We thank R. Colombo for helpful discussions regarding NMS-P715. We thank P.A. Eyeres for the kind gift of the phospho-MPS1 antibodies.

\section{References}

1. Gopalakrishnan V, Tao RH, Dobson T, Brugmann W and Khatua S: Medulloblastoma development: Tumor biology informs treatment decisions. CNS Oncol 4: 79-89, 2015. 
2. Fan X, Matsui W, Khaki L, Stearns D, Chun J, Li YM and Eberhart CG: Notch pathway inhibition depletes stem-like cells and blocks engraftment in embryonal brain tumors. Cancer Res 66: 7445-7452, 2006.

3. Wolter M, Reifenberger J, Sommer C, Ruzicka $T$ and Reifenberger G: Mutations in the human homologue of the Drosophila segment polarity gene patched $(\mathrm{PTCH})$ in sporadic basal cell carcinomas of the skin and primitive neuroectoderma tumors of the central nervous system. Cancer Res 57: 2581-2585, 1997.

4. Wodarz A and Nusse R: Mechanisms of Wnt signaling in development. Annu Rev Cell Dev Biol 14: 59-88, 1998.

5. Northcott PA, Korshunov A, Witt H, Hielscher T, Eberhart CG, Mack S, Bouffet E, Clifford SC, Hawkins CE, French P, et al: Medulloblastoma comprises four distinct molecular variants. J Clin Oncol 29: 1408-1414, 2011.

6. Cho YJ, Tsherniak A, Tamayo P, Santagata S, Ligon A, Greulich H, Berhoukim R, Amani V, Goumnerova L, Eberhart CG, et al: Integrative genomic analysis of medulloblastoma identifies a molecular subgroup that drives poor clinical outcome. J Clin Oncol 29: 1424-1430, 2011.

7. Taylor MD, Northcott PA, Korshunov A, Remke M, Cho YJ, Clifford SC, Eberhart CG, Parsons DW, Rutkowski S, Gajjar A, et al: Molecular subgroups of medulloblastoma: The current consensus. Acta Neuropathol 123: 465-472, 2012.

8. Leary SE and Olson JM: The molecular classification of medulloblastoma: Driving the next generation clinical trials. Curr Opin Pediatr 24: 33-39, 2012.

9. Harris PS, Venkataraman S, Alimova I, Birks DK, Balakrishnan I, Cristiano B, Donson AM, Dubuc AM, Taylor MD, Foreman NK, et al: Integrated genomic analysis identifies the mitotic checkpoint kinase WEE1 as a novel therapeutic target in medulloblastoma Mol Cancer 13: 72, 2014.

10. Liu X and Winey M: The MPS1 family of protein kinases. Annu Rev Biochem 81: 561-585, 2012.

11. Ling Y, Zhang X, Bai Y, Li P, Wei C, Song T, Zheng Z, Guan K, Zhang Y, Zhang B, et al: Overexpression of Mps1 in colon cancer cells attenuates the spindle assembly checkpoint and increases aneuploidy. Biochem Biophys Res Commun 450: 1690-1695, 2014.

12. Daniel J, Coulter J, Woo JH, Wilsbach K and Gabrielson E: High levels of the Mps1 checkpoint protein are protective of aneuploidy in breast cancer cells. Proc Natl Acad Sci USA 108 $5384-5389,2011$
13. Janssen A, Kops GJ and Medema RH: Targeting the mitotic checkpoint to kill tumor cells. Horm Cancer 2: 113-116, 2011

14. Colombo R, Caldarelli M, Mennecozzi M, Giorgini ML, Sola F, Cappella P, Perrera C, Depaolini SR, Rusconi L, Cucchi U, et al: Targeting the mitotic checkpoint for cancer therapy with NMS-P715, an inhibitor of MPS1 kinase. Cancer Res 70: 10255-10264, 2010.

15. Venkataraman S, Alimova I, Balakrishnan I, Harris P, Birks DK, Griesinger A, Amani V, Cristiano B, Remke M, Taylor MD, et al: Inhibition of BRD4 attenuates tumor cell self-renewal and suppresses stem cell signaling in MYC driven medulloblastoma. Oncotarget 5: 2355-2371, 2014.

16. Tyler RK, Chu ML, Johnson H, McKenzie EA, Gaskell SJ and Eyers PA: Phosphoregulation of human Mps1 kinase. Biochem J 417: 173-181, 2009.

17. Samkari A, White JC and Packer RJ: Medulloblastoma: Toward biologically based management. Semin Pediatr Neurol 22: 6-13, 2015.

18. Malumbres $\mathbf{M}$ and Barbacid $\mathrm{M}$ : Cell cycle kinases in cancer. Curr Opin Genet Dev 17: 60-65, 2007.

19. El-Sheikh A, Fan R, Birks D, Donson A, Foreman NK and Vibhakar R: Inhibition of Aurora Kinase A enhances chemosensitivity of medulloblastoma cell lines. Pediatr Blood Cancer 55: 35-41, 2010.

20. Harris PS, Venkataraman S, Alimova I, Birks DK, Donson AM, Knipstein J, Dubuc A, Taylor MD, Handler MH, Foreman NK, et al: Polo-like kinase 1 (PLK1) inhibition suppresses cell growth and enhances radiation sensitivity in medulloblastoma cells. BMC Cancer 12: 80, 2012.

21. Maachani UB, Kramp T, Hanson R, Zhao S, Celiku O, Shankavaram U, Colombo R, Caplen NJ, Camphausen K and Tandle A: Targeting MPS1 enhances radiosensitization of human glioblastoma by modulating DNA repair proteins. Mol Cancer Res 13: 852-862, 2015.

22. Wengner AM, Siemeister G, Koppitz M, Schulze V, Kosemund D, Klar U, Stoeckigt D, Neuhaus R, Lienau P, Bader B, et al: Novel Mps1 kinase inhibitors with potent antitumor activity. Mol Cancer Ther 15: 583-592, 2016.

23. Koch A, Maia A, Janssen A and Medema RH: Molecular basis underlying resistance to Mps1/TTK inhibitors. Oncogene 35: 2518-2528, 2016. 